\title{
Relationship between preoperative statins use and atrial fibrillation (AF) after cardiac surgery. analysis with a propensity score
}

\author{
M Fernandez-Zamora ${ }^{1}$, A Gordillo-Brenes ${ }^{2}$, MD Arias-Verdú1* ${ }^{*}$ E Curiel-Balsera ${ }^{1}$, A Herruzo-Aviles ${ }^{3}$, \\ M Garcia-Delgado ${ }^{4}$, JA Arboleda-Sánchez ${ }^{5}$, A Hinojosa-Perez ${ }^{6}$, R Rivera-Fernández ${ }^{1}$
}

From ESICM LIVES 2015

Berlin, Germany. 3-7 October 2015

\section{Objectives}

To analyze the relationship between preoperative statin use and the development of atrial fibrillation (AF) in postoperative cardiac surgery patients.

\section{Methods}

Prospective cohort study of cardiac surgery patients in 11 hospitals, included in the Spanish ARIAM register, during 2008-2012. Users of statins before surgery were matched to non-users according to a propensity score to quantify the probability of being treated with statins preoperatively, based on demographics, comorbidities, medication and type of surgery. We analyzed differences in the incidence of postoperative atrial fibrillation in both groups. The statistical study was done with the Student T, $\mathrm{X}^{2,}$ Mc-Nemar test, and logistic regression.

\section{Results}

$\mathrm{N}=7,276$ patients. Mean age: $63.91 \pm 1245$ years. Elective surgery: $85.9 \%$. Surgical risk EuroSCORE: $5.86 \pm 3.14$ points. ICU mortality: $7.6 \%$. Hospital mortality $10.1 \%$ (8.1\% missing).Before surgery, $51.5 \%$ of patients had taken statins and $25.8 \%$ had atrial fibrillation. After intervention, $21.9 \%$ presented atrial fibrillation as a complication during ICU stay.Among patients who were treated with statins preoperatively, AF occurred in $19.8 \%$ vs $22.5 \%$ in those no taking $(P=0.006)$. By multivariate analysis with logistic regression was observed that the frequency of AF as a complication to equal the other variables included in the model (prior history of AF, EuroSCORE, SAPS-3, urgent surgery and surgical valve type, time By-pass more than

${ }^{1}$ Hospital Regional Universitario Carlos Haya, Intensive Care Unit, Málaga, Spain Full list of author information is available at the end of the article
120 minutes) was not lower in patients taking statins preoperatively (OR: 0.975, CI: 0.861-1.105). After, we performed the analysis in the group of patients matched by propensity index to receive preoperatorive statins (1528 treated with statins and 1528 without statins) and we observed that the frequency of FA was $20.4 \%$ in patients treated with statins (1528) and $24.9 \%$ in 1528 untreated $(\mathrm{p}=0.003)$, OR: 0.772, CI: 0652-0916).Logistic regression performed in the group of patients matched by propensity score to receive preoperative statins (1528 treated with statins and 1528 without statins) was observed that the frequency of AF to equal the other variables included in the model (prior history of AF, SAPS-3, urgent surgery and surgical valve type, bypas time more than 120 minutes) was lower in patients taking statins preoperatively (OR: 0.779, CI: 0649-0936).

\section{Conclusions}

Preoperative statin use was associated with a lower risk of postoperative atrial fibrillation after cardiac surgery.

\section{Authors' details \\ ${ }^{1}$ Hospital Regional Universitario Carlos Haya, Intensive Care Unit, Málaga, Spain. ${ }^{2}$ Hospital Puerta Del Mar, Intensive Care Unit, Cádiz, Spain. ${ }^{3}$ Hospital Virgen del Rocio, Intensive Care Unit, Sevilla, Spain. ${ }^{4}$ Hospital Virgen de las Nieves, Granada, Spain. ${ }^{5}$ Hospital Regional Universitario Carlos Haya, Intensive Care Unit, Spain, Spain. ${ }^{6}$ Hopspital Virgen del Rocio, Intensive Care Unit, Sevilla, Spain.}

Published: 1 October 2015

\section{doi:10.1186/2197-425X-3-S1-A948}

Cite this article as: Fernandez-Zamora et al:: Relationship between preoperative statins use and atrial fibrillation (AF) after cardiac surgery. analysis with a propensity score. Intensive Care Medicine Experimental 2015 3(Suppl 1):A948.

\section{SpringerOpen ${ }^{\odot}$}

(0) 2015 Fernandez-Zamora et al.; This is an Open Access article distributed under the terms of the Creative Commons Attribution License (http://creativecommons.org/licenses/by/4.0), which permits unrestricted use, distribution, and reproduction in any medium, provided the original work is properly cited. 